\title{
Mecanismos comportamentales como predicción del uso de ad blocking en usuarios de noticias online
}

\author{
Behavioral mechanisms as prediction of the use of ad blocking \\ in online news users
}

\author{
Sánchez-Blanco, C., Sádaba, C. y Sanjurjo-Sanmartín, E. L. ${ }^{1}$ \\ Recibido: 22-02-2020 - Aceptado: 5-08-2020 \\ https://doi.org/10.26441/RC19.2-2020-A13
}

RESUMEN: Evitar contenidos comerciales durante el consumo de medios no es un fenómeno nuevo. La teoría reconoce distintos mecanismos para identificar esta conducta y el uso de bloqueadores de publicidad se puede reconocer como una respuesta comportamental activa y consciente a este tipo de contenido. Es interesante ahondar en los motivos que pueden explicar esta respuesta por parte del usuario. El presente artículo analiza en qué medida el uso de estos programas de bloqueo de publicidad está relacionado con actitudes conscientes y cuál es la probabilidad de que ciertos perfiles los usen más o menos. Para ello se estudia el caso de los usuarios de noticias online en España con los datos del Digital News Report 2018 que encuestó a una muestra representativa de 2023 españoles mayores de edad. A través de una regresión logística binomial, se demuestra que algunos aspectos demográficos como la edad y el género parecen indicar una mayor propensión a emplear este tipo de programas de bloqueo de publicidad online. También los usuarios con una actitud activa presentan más probabilidad de usar programas para bloquear la publicidad digital. Por contra, se muestra cómo hay dos perfiles de personas que tienen menor predisposición a usar estos programas: los que pagan por noticias online y los que tienen una mayor familiaridad y conocimiento crítico del sector de la información.

Palabras clave: publicidad digital; programas bloqueo publicidad; evitar publicidad; usuarios noticias online.

ABSTRACT: Avoiding commercial content while consuming media is not a new phenomenon. The theory recognizes different mechanisms to explain this behavior and the use of advertising blockers can be recognized as an active and conscious behavioral response to this type of content. It is interesting to delve into the reasons that can explain this response by the user. This article analyzes to what extent the use of this ad blocking software is related to conscious attitudes and what is the probability that certain profiles will use them more or less. The case of online news users in Spain is studied with the data from the Digital News Report 2018, which surveyed a representative sample of 2023 Spanish adults. Through a binomial logistic regression, it is shown that some demographic aspects such as age and gender seem to indicate a greater propensity to use this type of online advertising blocking software. Also users with an active attitude are more likely to use programs to block digital advertising. In contrast, it is shown how there are two profiles of people who are less likely to use these software: those who pay for online news and those who have greater familiarity and critical knowledge of the information sector.

Keywords: digital advertising; adblockers; advertising avoidance; online news users.

\footnotetext{
${ }^{1}$ Cristina Sánchez-Blanco es Doctora en Comunicación y Profesora Contratada Doctora del Departamento de Marketing y Empresas de Comunicación de la Facultad de Comunicación de la Universidad de Navarra. csblanco@unav.es, https://orcid.org/0000-0001-6263-6520
}

Charo Sádaba es Doctora en Comunicación y Profesora Titular del Departamento de Marketing y Empresas de Comunicación de la Facultad de Comunicación de la Universidad de Navarra. csadaba@unav.es, https://orcid. org/0000-0003-2596-2794

Elena-Luisa Sanjurjo-Sanmartín es Doctora en Economía Aplicada y profesora de probabilidad y estadística de la Facultad de Económicas de la Universidad de Navarra. esanjurjo@unav.es, https://orcid.org/0000-0002-9058-0883 


\section{Introducción y estado de la cuestión ${ }^{2}$}

El uso de los bloqueadores de publicidad digital supone una amenaza cierta a la industria publicitaria ya que permite con facilidad a los usuarios de tecnología acceder al contenido deseado sin mensajes comerciales. Según Global Web Index en 2018 el 47\% de los usuarios de internet en el mundo han usado algún tipo de software para bloquear contenidos publicitarios. El $42 \%$ de los usuarios son mujeres y el 58\% hombres; el $31 \%$ tienen entre 16 y 24 años, el $32 \%$ entre 25 y 35 años y el 20\% entre 35 y 44 años (Global Web Index, 2018). El último estudio de PageFair “2017 Adblock report" de enero de 2017, explica cómo el número global de dispositivos que bloquean anuncios ha aumentado considerablemente, de 142 millones en diciembre de 2015 a 615 millones en diciembre de 2016. Sólo en móviles, ha crecido de 108 a 380 millones en los mismos años mientras que en ordenadores ha pasado de 34 a 236 millones (Page Fair, 2017: 6; Sádaba y Sánchez-Blanco, 2019: 132).

En España, según el estudio más reciente sobre este tema "La percepción de la publicidad en TV e Internet 2018", elaborado por el Observatorio de la Publicidad (de la Asociación de Anunciantes), Imop y Wavemaker, "el 34\% de los internautas tiene instalado en alguno de sus navegadores programas para bloquear la publicidad. En concreto, un 33\% los instala en el PC/ordenador, un 6,1\% en la tablet y un $7 \%$ en su smartphone, siendo el perfil de estos usuarios más masculino y joven (generación Z y millennials)" (2018: 45).

Lo que está claro es que el avance de la tecnología ha facilitado que los usuarios digitales incrementen el control sobre el contenido y la publicidad que consultan y eviten el no deseado (Johnson, 2013: 128; Seyedghorban, Tahernejad y Matanda, 2016: 125) lo que pone en alerta a las empresas y a los medios de comunicación que ven peligrar sus inversiones (McDonald, 2018: 76) y una de sus principales vías de ingresos y que exigen una reflexión profunda de cómo debe ser la publicidad online para que conecte con los consumidores y no se rechace. Recientemente, Lancelot, Cases y Russell (2019) y Chinchanachokchai y De Gregorio (2020) lo han estudiado en concreto sobre la publicidad en redes sociales. Los datos apuntan a que son los más jóvenes los que hacen un uso más intensivo de estos bloqueadores (Southgate, 2017: 233). Pese al evidente interés de este fenómeno, por su clara incidencia sobre el negocio de los medios de comunicación, hay pocos trabajos académicos sobre el tema que, en la mayor parte de los casos, queda atendido solo por publicaciones profesionales. Una de las posibles perspectivas para analizarlo es la del ad avoidance (evitar publicidad): el estudio de las motivaciones y comportamientos por los que los usuarios de los medios tienden a rechazar el contenido comercial.

Speck y Elliott definen evitar publicidad como todas las acciones que los usuarios de los medios realizan para reducir su exposición al contenido publicitario (Speck y Elliott, 1997: 61). En el caso de internet, evitar la publicidad (Söllner y Dost, 2019: 303) adquiere una relevancia especial ya que se trata de un medio al que se accede con un fin mucho más concreto que a otros medios, por lo que la publicidad suele percibirse como más intrusiva (Cho y Cheon, 2004; Korgaonkar y Wolin, 1999; Goodrich, Schiller y Galletta, 2015; Li, Edwards y Lee, 2002; Campbell, Thomson, Grimm y Robson, 2017). De hecho, Edwards, Li y Lee (2002: 94) demostraron que la sensación de intrusividad de la publicidad en internet es precursora de los sentimientos de irritación y de comportamientos que llevan a evitar la publicidad.

Varios autores explican que evitar el contenido comercial tiene tres manifestaciones concretas: la cognitiva, la afectiva y la comportamental (Li, Edwards y Lee, 2002; Prendergast, Tsang y Cheng,

\footnotetext{
2 Investigación financiada por el proyecto "Usos y preferencias informativas en el nuevo mapa de medios en España: audiencias, empresas, contenidos y gestión de la reputación en un entorno multipantalla" cofinanciado por Ministerio de Economía y Competitividad y el Fondo Europeo de Desarrollo Regional (CSO2015-64662-C4-1-R).
} 
2014; Cho y Cheon, 2004). Los mecanismos cognitivos para evitar el contenido publicitario son respuestas psicológicas que llevan a ignorarlo deliberadamente, pese a estar en contacto con él (Prendergast, Tsang y Cheng, 2014). El bloqueo afectivo, por su parte, implica la aparición de emociones o sentimientos negativos ante el contenido publicitario, tales como el enfado o la repulsa (Alwitt y Prabhaker, 1994; Phillips y Noble, 2007). Por último, los mecanismos comportamentales implican que el usuario lleva a cabo alguna acción que busca eliminar la publicidad de su situación de consumo (Cho y Cheon, 2004: 91), tales como cambiar de canal de televisión o instalar un software que bloquee el contenido publicitario online (Baek y Morimoto, 2012; Lancelot, Cases y Russell, 2019: 416), que es el caso pertinente en este estudio.

En algunos de los estudios realizados para analizar quiénes y por qué se evita la publicidad en los medios, se apreció que variables como la edad, el género o el nivel educativo podrían ser relevantes para entender los perfiles y las razones que llevaban a los usuarios a evitar la publicidad (Prendergast, Tsang y Cheng, 2014: 134). Speck y Elliott (1997) demostraron que en medios impresos los jóvenes evitaban publicidad más que los usuarios mayores, al igual que pasaba en la televisión, según Danaher (1995). Por su parte, Rojas-Méndez, Davies y Canan (2009) apuntaban que las mujeres y las personas con mayor nivel educativo tendían a adoptar comportamientos para evitar la publicidad en televisión. Sin embargo, Van der Goot, Rozendaal, Opree, Ketelaar y Smit (2018: 301) llevaron a cabo un estudio en seis países sobre las actitudes ante la publicidad y si varias generaciones la evitaban en cinco medios diferentes: la web, las redes sociales, los teléfonos móviles, los periódicos y la televisión y concluyeron que no había diferencias por edades. En este sentido, es interesante seguir ahondando, como apuntan otros autores como Li, Wang y Zhang (2020, p. 1074) en estos estudios para intentar dilucidar si la edad o el género son variables relevantes en esta temática.

Estos comportamientos que buscan evitar la publicidad, siguiendo a Tang, Zhang y Fei Wu (2015: 513-514), pueden ser activos o pasivos. En concreto, la descarga de software para bloquear la publicidad encaja en la tipología de comportamiento activo. Se trata de un comportamiento concreto y consciente, al igual que otras acciones menos determinantes como el scroll a través de la página o abandonar una página concreta ante la presencia de anuncios (Cho y Cheon, 2004; Baek y Morimoto, 2012; Seyedghorban, Tahernejad y Matanda, 2016: 121).

Entre las razones principales que llevan a la descarga de este tipo de software pueden identificarse algunas subjetivas y otras objetivas (Sádaba y Sánchez-Blanco, 2018: 127). Entre las primeras, Tudoran destaca la percepción de intrusión como una de las principales causas (2019: 159). Esta autora (2019: 147), además, señala que el comportamiento está determinado por los conocimientos, pero también por los sentimientos y por las emociones que genera este contenido, ideas tratadas anteriormente por Eagly y Chaiken (1998). Otros autores sin embargo (Singh y Potdar, 2009; Walbesser, 2011; Nithyanand et. al, 2016) apuntan a razones más objetivas como la excesiva cantidad de publicidad que molesta e impide la navegación; la búsqueda de la privacidad (Barbacovi, 2017: 274); y otras relacionadas con prevenir el malware, evitar que el contenido comercial consuma el ancho de banda contratado o la mayor incomodidad en la visualización del contenido en los dispositivos móviles.

Cho y Chan ya en 2004 destacaban la relevancia de diferenciar los perfiles de usuarios a la hora de evitar los contenidos comerciales. Cabe pensar que, en el caso concreto de los consumidores de información online, quienes buscan una información específica en un momento concreto tengan diferentes razones para evitar la publicidad en Internet entre las que podrían estar la presión del tiempo, la publicidad irrelevante o que no está dirigida a ellos, diferentes a las de quienes están navegando por mero entretenimiento o distracción y que perciben que los anuncios no son divertidos (2004, p. 94). Recientemente se ha comprobado, por ejemplo, que los usuarios tienen una predisposición 
positiva a apagar los bloqueadores de anuncios si los medios de comunicación les hacen algún tipo de llamamiento explicando el sentido y la necesidad de la publicidad para ese medio (Söllner; Dost, 2019: 309).

Entendiendo, por tanto, que la descarga y el uso de bloqueadores de publicidad puede ser calificado como un mecanismo comportamental consciente y activo para evitar el contenido comercial, el objetivo de este artículo es analizar en qué medida su uso está relacionado con ciertas características y comportamientos de los usuarios y cuál es la probabilidad de que ciertos perfiles de usuarios usen más o menos los programas de bloqueo de anuncios. Parece especialmente pertinente analizar la conducta y la motivación de un grupo de usuarios en un contexto de consumo de contenido limitado, en este caso la información, ya que eso apunta a un propósito claro en la navegación frente al cual valorar su interacción con otros contenidos. Por tanto, las preguntas de investigación planteadas son las siguientes:

- P1. ¿Hay diferencias significativas por edad, género y nivel de ingresos en el uso de programas de bloqueo de publicidad online?

- P2. ¿Los usuarios con mayor frecuencia de acceso a las noticias online tienen mayor predisposición para usar bloqueadores de anuncios?

- P3. ¿Los usuarios con un grado alto de alfabetización informativa bloquean la publicidad más que los que tienen menor grado?

- P4. ¿Una actitud proactiva para gestionar el tiempo cuando se consultan noticias online, en concreto a través de redes sociales, tiene relación directa con el uso de los bloqueadores de publicidad?

- P5. ¿Los usuarios que más bloquean publicidad son los que más pagan por los contenidos online?

\section{Metodología}

El modelo aplicado mediante regresión logística binaria analiza cuánto más probable es la manifestación concreta de evitar la publicidad mediante el uso de software de bloqueo de publicidad en internet en los usuarios de noticias online, teniendo en cuenta cuatro dimensiones que corresponden a varios comportamientos conscientes concretos de su consumo digital de noticias. La primera dimensión es sociodemográfica (pregunta de investigación 1) y analiza la probabilidad de usar programas de bloqueo publicitario en función del género, la edad (inferior o superior al umbral de los 35 años) o el nivel de ingresos. La segunda dimensión, que incluye las preguntas de investigación 2 y 4, tiene en cuenta el tiempo en dos de sus vertientes: frecuencia de acceso a internet y la actitud proactiva para gestionar el tiempo cuando se consultan noticias online, en concreto en redes sociales. La tercera dimensión aborda el grado de conocimiento del mundo de los medios y la valoración de la publicidad como su fuente de financiación (pregunta de investigación 3). Por último, la cuarta dimensión aborda la disponibilidad o no de pagar por noticias en internet (pregunta de investigación 5).

Los datos utilizados provienen, como ya se ha comentado, del Digital News Report 2018 (Reuters Institute for the Study of Journalism, Universidad de Oxford). Se trata del estudio más comprehensivo que se realiza sobre el consumo de noticias online. Se lleva a cabo anualmente a través de una encuesta online, respondida exclusivamente a través de ordenador, y realizada por YouGov en 37 países con muestras nacionales en torno a 2000 usuarios (en España, para el año $2018, n=2023$ con una población de 46 millones y una penetración de internet del 87\%). Todos 
los participantes son internautas adultos que han consumido noticias en el último mes, representativos de la población conectada según criterios sociodemográficos y geográficos (Digital News Report, 2018: 5-6).

\subsection{Variable dependiente}

El uso de adblockers en internet ha sido medido como una variable dicotómica y codificada con valor 1 en el caso de que "sí he descargado software" y 0 en el caso de no hacerlo. En la encuesta es la pregunta directa: “¿Has descargado algún programa de bloqueo de publicidad en alguno de tus dispositivos personales que te permitan bloquear publicidad en Internet (por ejemplo Adblock Plus)?" (QAD2).

Los datos del Digital News Report 2018 recogidos en ordenador, arrojan que un 34,\% de los usuarios de noticias online alguna vez se ha instalado algún tipo de programa para bloquear anuncios. Es relevante detallar, a través de las siguientes tablas (1 y 2), por género y por edad, el uso de estos programas de bloqueo.

Tabla 1. Tabla de frecuencias de uso de bloqueadores de publicidad online por género

\begin{tabular}{|c|c|c|c|c|}
\hline & & \multicolumn{2}{|c|}{ Género } & \multirow{2}{*}{ Total } \\
\hline & & Hombre & Mujer & \\
\hline \multirow{5}{*}{ QAD2 } & \multirow{2}{*}{ Sí } & 390 & 309 & 699 \\
\hline & & $40,8 \%$ & $32,3 \%$ & $36,5 \%$ \\
\hline & \multirow{2}{*}{ No } & 567 & 648 & 1215 \\
\hline & & $59,2 \%$ & $67,7 \%$ & $63,5 \%$ \\
\hline & Total & 957 & 957 & 1914 \\
\hline
\end{tabular}

Fuente: elaboración propia

Tabla 2. Tabla de frecuencias de uso de bloqueadores de publicidad online por edad

\begin{tabular}{|c|c|c|c|c|c|c|c|}
\hline & & \multicolumn{5}{|c|}{ Edad } & \multirow{2}{*}{ Total } \\
\hline & & $18-24$ & $25-34$ & $35-44$ & $45-54$ & $55+$ & \\
\hline \multirow{4}{*}{ QAD2 } & \multirow{2}{*}{ Sí } & 110 & 149 & 123 & 120 & 197 & 699 \\
\hline & & $67,5 \%$ & $52,8 \%$ & $31,6 \%$ & $32,2 \%$ & $27,9 \%$ & $36,5 \%$ \\
\hline & \multirow{2}{*}{ No } & 53 & 133 & 266 & 253 & 510 & 1215 \\
\hline & & $32,5 \%$ & $47,2 \%$ & $68,4 \%$ & $67,8 \%$ & $72,1 \%$ & $63,5 \%$ \\
\hline & Total & 163 & 282 & 389 & 373 & 707 & 1914 \\
\hline
\end{tabular}

Fuente: elaboración propia

Se demuestra que los usuarios que más bloquean publicidad online son los más jóvenes, sobre todo las franjas de edad 18-24 y 25-34 años y algo más los hombres que las mujeres.

\subsection{Variables independientes}

Las variables explicativas del modelo se pueden agrupar en cinco bloques, que corresponden a cada una de las preguntas de investigación. 
En el caso de las variables sociodemográficas (pregunta de investigación 1), el "género", se ha codificado como variable dicotómica ( $1=$ hombre; $2=$ mujer); la "edad" como variable dicotómica ( $1=$ Por debajo de los 35 años; $0=$ igual o mayor a 35 años) y el "nivel de ingresos" como variable continua agrupada por intervalos.

La frecuencia de acceso a internet y de consulta de noticias online (pregunta de investigación 2) se han codificado como variables categóricas politómicas con rango decreciente de 1 a 10.

El grado de conocimiento sobre medios (pregunta de investigación 3) se ha analizado a partir de cuatro preguntas presentes en el cuestionario, a través de las cuales se puede valorar si los usuarios son conscientes del funcionamiento de los medios:

- dependencia del medio ante la publicidad

- quién es el responsable de escribir una nota de prensa

- decisión sobre qué noticias se publican

- y la combinación de las tres anteriores

En todas ellas, sólo una respuesta era la correcta y de respuesta única. En este caso se realizó un recuento del número de preguntas acertadas.

Respecto a la optimización del tiempo invertido en la búsqueda de información en redes sociales (pregunta de investigación 4), se han tenido en cuenta cuatro preguntas que indagan en los criterios que valoran la importancia de la información que se pretende leer en redes sociales. Se pregunta sobre si la decisión de consultar una noticia a través de las redes sociales y la conciencia de que ha merecido la pena depende de la marca del medio; del titular o la imagen; de la persona que comparte la información o del número de likes y comentarios de la noticia. Todas las variables son categóricas y politómicas con rango decreciente de 1 a 5 .

Por último, el pago por contenido (pregunta de investigación 5) hace referencia a la pregunta sobre si se ha pagado por noticias online en el último año, como variable dicotómica (1=Sí y $0=\mathrm{No})$.

\section{Resultados}

\subsection{Análisis exploratorio de datos}

La muestra representativa de 2023 personas de nacionalidad española está formada por un 48,9\% de hombres y un $51,1 \%$ de mujeres, con un $22,7 \%$ de edades por debajo de los 35 años. La Tabla 3 presenta las frecuencias de respuesta dadas a las variables: dependiente (QAD2) e independientes del modelo: Age_rc08; gender_int; income_Spain; Q14_d; Q7a y Q12C_2018_Q12C_2018_1 a Q12C_2018_4; Q1_aNEW y Q1_bNEW 
Tabla 3. Frecuencias de respuesta para las variables dependiente e independientes del modelo (\%)

\begin{tabular}{|c|c|}
\hline $\begin{array}{l}\text { DESCARGA SOFTWARE BLOQUEO PUBLICIDAD } \\
\text { ¿Se ha descargado software o similar que le permita bloquear anuncios en } \\
\text { internet? (QAD2) }\end{array}$ & $\begin{array}{l}\text { Sí } 36,5 \\
\text { No } 63,5\end{array}$ \\
\hline \multicolumn{2}{|l|}{ SOCIODEMOGRÁFICAS } \\
\hline Edad hasta 35 (Age_rc08) & $\begin{array}{l}\text { No } 77,3 \\
\text { Sí } 22,7\end{array}$ \\
\hline Género (gender int) & \begin{tabular}{|l|} 
Hombre 48,9 \\
Mujer 51,1
\end{tabular} \\
\hline Nivel de ingresos (income_Spain) & 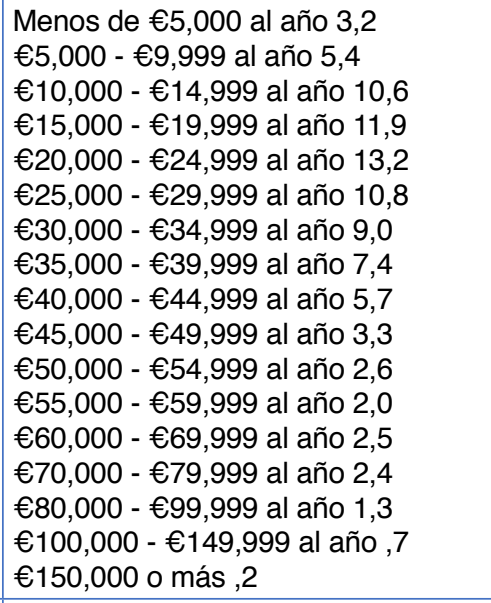 \\
\hline FRECUENCIA USO DE INTERNET & $\begin{array}{l}\text { Internet (Q1_aNEW) } \\
\text { Más de } 10 \text { veces al día } 45,7 \\
\text { Entre } 6 \text { y } 10 \text { veces al día } 20,5 \\
\text { Entre } 2 \text { y } 5 \text { veces al día } 26,1 \\
\text { Una vez al día } 6,0 \\
4-6 \text { días por semana }, 7 \\
\text { 2-3 días por semana }, 5 \\
\text { Una vez por semana }, 1 \\
\text { Menos de una vez por semana ,1 }\end{array}$ \\
\hline FRECUENCIA USO DE NOTICIAS & $\begin{array}{l}\text { Noticias (Q1b_NEW) } \\
\text { Más de } 10 \text { veces al día } 45,7 \\
\text { Entre } 6 \text { y } 10 \text { veces al día } 20,5 \\
\text { Entre } 2 \text { y } 5 \text { veces al día } 26,1 \\
\text { Una vez al día } 6,0 \\
4-6 \text { días por semana }, 7 \\
\text { 2-3 días por semana }, 5 \\
\text { Una vez por semana }, 1 \\
\text { Menos de una vez por semana }, 1\end{array}$ \\
\hline $\begin{array}{l}\text { CONOCIMIENTO DE LOS MEDIOS } \\
\text { ¿Qué sentencia describe mejor la situación financiera de la mayoría de los diarios; } \\
\text { sitios webs y apps? Por favor seleccione una solamente. (Q14d) }\end{array}$ & $\begin{array}{l}\text { La mayoría obtiene beneficios } 51,4 \\
\text { La mayoría cubren costes } 32,7 \\
\text { La mayoría obtienen pérdidas } \\
\text { (Correcta) } 15,9\end{array}$ \\
\hline $\begin{array}{l}\text { OPTIMIZACIÓN DEL TIEMPO NOTICIAS EN REDES SOCIALES } \\
\text { Indique su nivel de acuerdo: Al mirar historias en las redes sociales, la marca de } \\
\text { noticias es muy importante para ayudarme a decidir si la información valdrá la } \\
\text { pena. (Q12c_1) }\end{array}$ & $\begin{array}{l}\text { Muy en desacuerdo } 3,6 \\
\text { Algo en desacuerdo } 4,1 \\
\text { Ni de acuerdo ni en desacuerdo } 24,8 \\
\text { Algo de acuerdo } 38,0 \\
\text { Muy de acuerdo } 29,5\end{array}$ \\
\hline $\begin{array}{l}\text { Indique su nivel de acuerdo: Cuando veo historias en las redes sociales, el titular } \\
\text { o la imagen son muy importantes para ayudarme a decidir si la información valdrá } \\
\text { la pena. (Q12c_2) }\end{array}$ & $\begin{array}{l}\text { Muy en desacuerdo } 4,4 \\
\text { Algo en desacuerdo } 7,3 \\
\text { Ni de acuerdo ni en desacuerdo } 31,5 \\
\text { Algo de acuerdo } 41,7 \\
\text { Muy de acuerdo } 15,0\end{array}$ \\
\hline $\begin{array}{l}\text { Indique su nivel de acuerdo: Al mirar las historias en las redes sociales, la } \\
\text { persona que compartió la historia es muy importante para ayudarme a decidir si la } \\
\text { información valdrá la pena. (Q12c_3) }\end{array}$ & $\begin{array}{l}\text { Muy en desacuerdo } 5,5 \\
\text { Algo en desacuerdo } 8,0 \\
\text { Ni de acuerdo ni en desacuerdo } 29,6 \\
\text { Algo de acuerdo } 38,9 \\
\text { Muy de acuerdo } 18,0\end{array}$ \\
\hline
\end{tabular}


Indique su nivel de acuerdo: Al mirar las historias en las redes sociales, la
cantidad de comentarios como los "likes" de las acciones es muy importante para
ayudarme a decidir si la información valdrá la pena. (Q12c_4)

PAGO DE NOTICIAS

¿Ha pagado por contenido ONLINE de noticias o accedido a pagar por un servicio

en el último año? (suscripción digital, o combinada con impresa, o artículo; app o

e-edición) (Q7a)
Muy en desacuerdo 17,0

Algo en desacuerdo 16,6

Ni de acuerdo ni en desacuerdo 35,2

Algo de acuerdo 24,2

Muy de acuerdo 6,9

Sí 11,3

No 88,7

Fuente: elaboración propia

\subsection{Regresión logística}

Los resultados de la modelización explican qué personas con determinados comportamientos conscientes y determinadas características: sociodemográficas; con una frecuencia de uso de internet y de noticias; con grado de conocimiento sobre los medios y su financiación; que no quiere perder tiempo en la búsqueda de información por internet, en concreto en redes sociales; y además están dispuestas a pagar por información presentan una mayor probabilidad de que se instalen un bloqueador para anuncios.

En el modelo 1 se incluyen solo las variables sociodemográficas; en el 2 el tiempo de uso, y la disposición al pago; el cuarto modelo incorpora la optimización del tiempo de búsqueda y el último modelo aborda el grado de conocimiento del mundo de los medios o la valoración de la publicidad como fuente de financiación.

Tabla 4. Modelo de regresión binomial

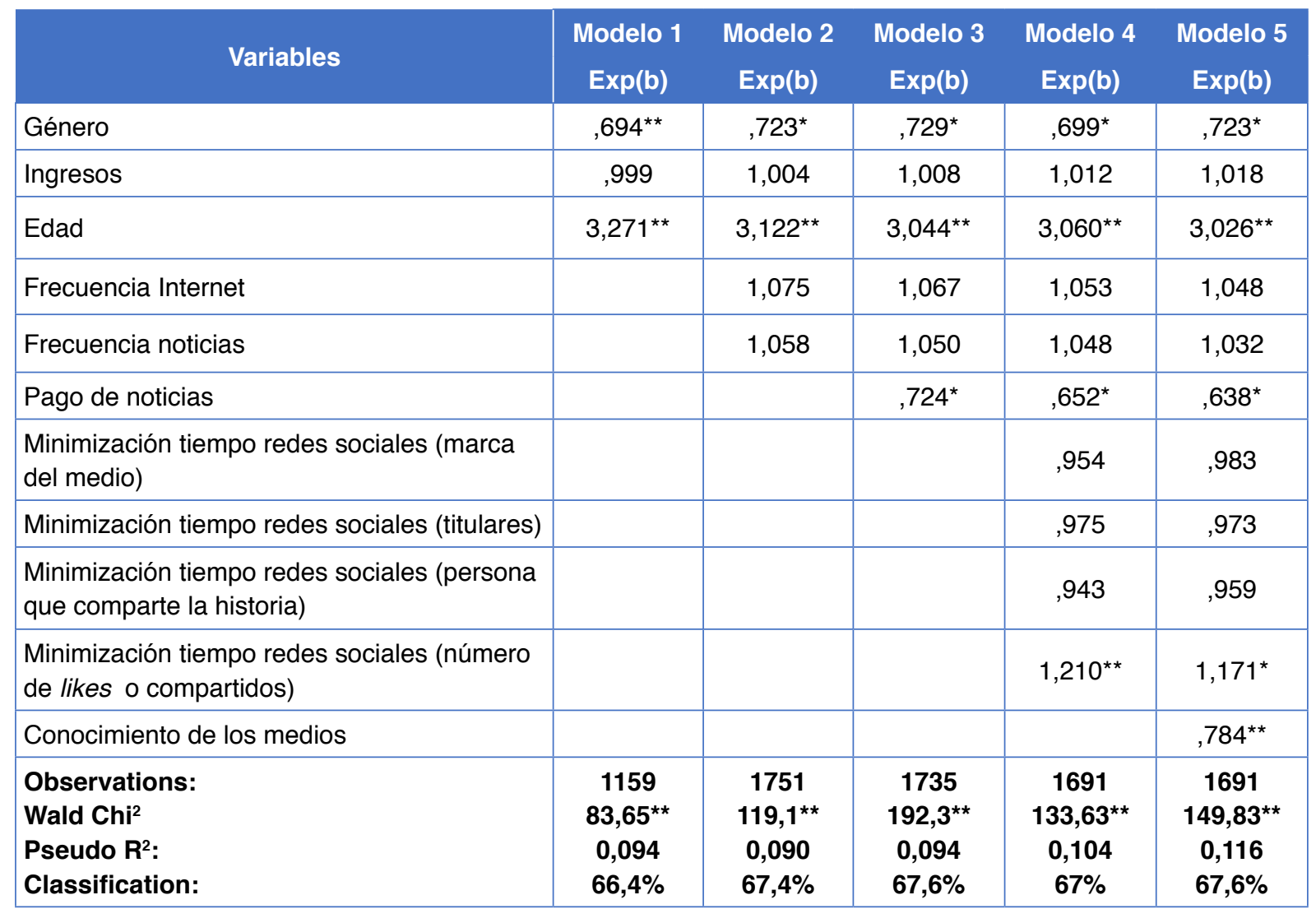

${ }^{*} p<.05 ;{ }^{* *} p<.01$ Última categoría en las variables categóricas es la que se ha usado como referencia Fuente: elaboración propia 
A través de las exponenciales de los coeficientes estimados en el modelo, se analiza cuántas veces es más probable que se produzca la descarga de software para bloquear anuncios. De la tabla 4 es posible concluir algunos resultados.

En cuanto a la primera pregunta de investigación, la posibilidad de instalar un programa para bloquear anuncios es un 30\% menor en mujeres que en hombres y hasta 3 veces más probable si la edad es menor que 35 años. Los ingresos no suponen un factor explicativo; así como tampoco la regularidad $\mathrm{y}$ frecuencia de acceso a internet y a noticias, que da respuesta a la segunda pregunta de investigación.

Sin embargo, en lo que se refiere a la tercera pregunta de investigación, una mayor cultura de medios y su funcionamiento provoca una disminución del uso de los bloqueadores de publicidad online de hasta un $22 \%$ respecto a quienes no tienen ese conocimiento.

Una actitud proactiva para gestionar el tiempo cuando se consultan noticias online, en concreto a través de redes sociales, (cuarta pregunta de investigación), aumenta hasta 1,2 veces más la posibilidad de que se use bloqueadores.

Por último, en lo que se refiere a la quinta pregunta de investigación, se aprecia cómo el pago por noticias reduce un $36 \%$ el uso de bloqueadores de publicidad online.

\section{Discusión}

Evitar la publicidad, como se ha entendido en el marco teórico, en internet es más relevante si cabe que en otros medios ya que internet es un medio al que se accede con un fin mucho más concreto, por lo que la publicidad suele percibirse como más intrusiva. Teniendo en cuenta la mayor fragmentación de la inversión publicitaria y el impacto negativo que esta ha tenido sobre las cuentas de resultados de los medios de comunicación, parece razonable seguir ahondando en las razones que llevan a los usuarios a evitar la publicidad. Conocer los motivos y los comportamientos asociados que llevan a los usuarios a bloquear los contenidos comerciales puede ayudar a diseñar estrategias de mitigación que aseguren la sostenibilidad de los medios, en un entorno en el que la tecnología facilita cada vez más formas para que los usuarios eviten, de manera consciente, el contenido comercial no deseado.

La investigación realizada pone de manifiesto que los mecanismos para evitar publicidad tienen explicaciones diversas. Como se había explicado en el marco teórico no hay un consenso sobre si la edad, el género y los ingresos son variables decisivas para analizar los perfiles de personas que evitan más la publicidad. Se había indicado que se veía relevante seguir investigando en esta línea y en este estudio, con sus limitaciones concretas, se ha demostrado que hay algunos aspectos demográficos como la edad y el género que parecen indicar una mayor propensión a evitar los contenidos comerciales en el consumo de información online a través de los bloqueadores de anuncios. Parece que son los usuarios más jóvenes y hombres los que usan más los bloqueadores de publicidad online. Esta idea quedaba apuntada en varios estudios profesionales, y es relevante seguir profundizando en otras cuestiones de índole más subjetiva. En este punto cabe solo añadir que es particularmente interesante que sean los públicos de menor edad los más dispuestos a usar este tipo de sistemas para bloquear el contenido comercial, por lo que puede indicar de tendencia a futuro a la que habrá que seguir prestando atención.

No se aprecia relación entre la frecuencia de acceso a internet y a las noticias y el uso de los bloqueadores, pero el estudio empírico realizado sí muestra que ciertos comportamientos de los usuarios de noticias online españoles guardan relación con una menor o mayor predisposición a usar programas de bloqueo de anuncios. Conocerlos podrá facilitar a la industria tomar medidas más específicas y más eficaces para asegurar el impacto publicitario que medios y marcas necesitan. 
En cuanto al pago, es razonable que las personas que pagan habitualmente por noticias online tengan menor predisposición a evitar los mensajes comerciales a través de un programa de bloqueo de la publicidad ya que, de hecho, ese pago por las noticias supone precisamente una reducción de la publicidad que reciben.

Así, la relación más importante se encuentra en los perfiles de personas que pagan por noticias online y también los que tienen una mayor familiaridad y conocimiento crítico del sector de la información, ya que esas actitudes y comportamientos hacen que disminuya la propensión a utilizar bloqueadores. En cuanto al conocimiento de los medios también se entiende porque, si se es más consciente de cómo se sostiene un medio de comunicación, el usuario tiene más reparo en bloquear y se acepta mejor la publicidad. Esto es consistente con el hallazgo de Söllner y Dost (2019: 309) cuando confirman que un mensaje advirtiendo a los visitantes de las consecuencias negativas para el medio de usar bloqueadores lleva consigo un menor uso de estas herramientas para evitar publicidad.

Y, por otro lado, vemos que ciertos perfiles, aunque en menor medida, presentan más probabilidad de usar programas para bloquear la publicidad digital. Se trata de usuarios con una actitud activa y que buscan aprovechar al máximo el tiempo invertido en la consulta de noticias online en concreto a través de redes sociales y los que dan mucha importancia al número de likes o comentarios a la hora de decidir si merece la pena consultar las noticias. Este usuario más enfocado y más práctico no deja de ser un perfil muy atractivo dado su vínculo con el contenido que consume, pero el estudio muestra que tiene mayor predisposición a bloquear la publicidad a través de programas concretos. Se ve una relación interesante entre su actitud activa ante el consumo de noticias y a la vez ese comportamiento activo y consciente de evitar la publicidad, como se analizaba en el marco teórico, que es el bloqueo de publicidad online a través de programas específicos. Conocer más cómo y en qué condiciones el contenido comercial podría apelar a su sentido más práctico abre nuevas vías de investigación con interés para marcas y medios.

Son interesantes las iniciativas que se están desarrollando desde la industria, como Coalition for Better Ads. En España IAB Spain ha trabajado recientemente la "Guía para mejorar la experiencia del usuario con la publicidad digital" (2019). Con las limitaciones propias de este único estudio, lo que sí se destaca es que es relevante analizar en profundidad las actitudes y los comportamientos de los usuarios online para poder, desde la industria publicitaria, ofrecer una experiencia de navegación más centrada en las necesidades de los usuarios que también sea beneficiosa para los medios de comunicación.

El estudio se ha realizado sobre una muestra concreta de usuarios de noticias online en España. Esto ha permitido asegurar un cierto foco en la navegación que permite entender algunos de los resultados cuando estos usuarios se encuentran con contenidos comerciales. No obstante, es también una limitación del estudio que debería ser replicado en otros contextos de uso, por ejemplo, entre quienes buscan entretenimiento, o satisfacer necesidades de comunicación, para entender si las pautas de uso se repiten o varían.

\section{Bibliografía}

Alwitt, L. F. y Prabhaker, P. R. (1994). Identifying who dislikes television advertising: Not by demographics alone. Journal of Advertising Research, 34 (6), pp. 17-29.

Asociación de Anunciantes (2018). La percepción de la Publicidad en tv e Internet 2018. https:// www.anunciantes.com/estudio-aea/percepcion-del-consumidor.pdf

Baek, T. H. y Morimoto, M. (2012). Stay away from me. Journal of Advertising, 41, 1, pp. 59-76. https://doi.org/10.2753/JOA0091-3367410105 
Campbell, C., Mattison Thompson, F., Grimm, P. E. y Robson, K. (2017). Understanding Why Consumers Don't Skip Pre-Roll Video Ads. Journal of Advertising, 46, 3, pp. 411-423. https://doi. org/10.1080/00913367.2017.1334249

Chinchanachokchai, S. y De Gregorio, F. (2020). A consumer socialization approach to understanding advertising avoidance on social media. Journal of Business Research, 110, pp. 474483. https://doi.org/10.1016/j.jbusres.2020.01.062

Cho, C. y Cheon, H. J. (2004). Why do People Avoid Advertising on the Internet? Journal of Advertising, 33, 4, pp. 89-97. http://dx.doi.org/10.1080/00913367.2004.10639175

Digital News Report (2018). Digital news report 2018. Resumen ejecutivo, http://www. digitalnewsreport.es/introduccion-digitalnewsreport-es-2018-una-audiencia-diversa-ypreocupada-por-la-desinformacion/

Danaher, P. (1995). What happens to television ratings during commercial breaks?Journal of Advertising Research, 35, 1, pp. 37-47.

Eagly, A. H. y Chaiken, S. (1998). Attitude structure and function. En Gilbert, D. T.; Fiske, S.T. y Lindzey, G. (Eds), The Handbook of Social Psychology, McGraw-Hill, New York, NY, pp. 269322.

Edwards, S.M.; Li, H. y Lee, J-H. (2002). Forced Exposure and Psychological Reactance: Antecedents and Consequences of the Perceived Intrusiveness of Pop-Up Ads. Journal of Advertising, 31, 3, pp. 83-95, DOI: 10.1080/00913367.2002.10673678

Global Web Index (2018). The digital trends to watch 2018, https://www.globalwebindex.com/ reports/trends-18

Goodrich, K., Schiller, S. Z. y Galletta, D. (2015). Consumer reactions to intrusiveness of onlinevideo advertisements: do length, informativeness, and humor help (or hinder) marketing outcomes? Journal of Advertising Research, 55, 1, pp. 37-50. http://dx.doi.org/10.2501/JAR-55-1-037-050

IAB Spain (2019). Guía para mejorar la experiencia del usuario con la publicidad digital. https:// iabspain.es/wp-content/uploads/gua-para-mejorar-la-experiencia-del-usuario-con-la-publicidaddigital.pdf

Johnson, J. P. (2013). Targeted Advertising and Advertising Avoidance. RAND Journal of Economics, 44, 1, pp. 128-44. https://doi.org/10.1111/1756-2171.12014

Korgaonkar, P. K.; Wolin, L.D. (1999). A Multivariate Analysis of Web Usage. Journal of Advertising Research, 39, 2, pp. 53-68.

Lancelot, C., Cases, A-S., Russell, C. (2019). Consumers' Responses to Facebook Advertising across PCs and Mobile Phones. Journal of Advertising Research, 59, 4, pp. 414-432. DOI: 10.2501/JAR-2019-029

Li, H.; Edwards, S.M. y Lee, J.H. (2002). Measuring the Intrusiveness of Advertisements: Scale Development and Validation. Journal of Advertising, 31, 2, pp. 37-47. https://doi.org/10.1080/009 13367.2002.10673665

Li, X., Wang, C. y Zhang, Y. (2020). The dilemma of social commerce: Why customers avoid peer-generated advertisements in mobile social networks. Internet Research, 30, 3, pp. 1059-1080. https://doi.org/10.1108/INTR-02-2017-0045 
McDonald, S. C. (2018). What Do We Really Know about Attitudes Toward Privacy and Advertisement Avoidance? Journal of Advertising Research, 58, 1, pp. 75-76. doi:10.2501/JAR2018-010

Nithyanand, R., Khattak, S., Javed, M., Vallina-Rodriguez, N., Falahrastegar, M., Powles, J. E., De Cristofaro, E., Haddadi, H. y Murdoch, S. J. (2016). Adblocking and counter-blocking: a slice of the arms race. En: Proceedings of the 6th USENIX Workshop on Free and Open Communications on the Internet, FOCI 2016, Austin, TX, August.

Page Fair (2017). 2017 Adblock report, https://pagefair.com/blog/2017/adblockreport/

Phillips, J., Noble, S. M. (2007). Simply Captivating: Understanding Consumers' Attitudes toward the Cinema as an Advertising Medium. Journal of Advertising, 36, 81-94. http://dx.doi. org/10.2753/JOA0091-3367360106

Prendergast, G. P., Tsang, A.S.L. y Cheng, R. (2014). Predicting Handbill Avoidance in Hong Kong and the UK. European Journal of Marketing, 48, 1/2, pp. 132-46. http://dx.doi. org/10.1108/EJM-05-2011-0244

Rojas-Méndez, J.I., Davies, G. y Canan, M. (2009). Universal differences in advertising avoidance behavior: a cross-cultural study. Journal of Business Research, 62, 10, pp. 947-954.

Sádaba, C. y Sánchez-Blanco, C. (2018). El uso de bloqueadores de publicidad entre los usuarios de noticias online en españa: perfiles y motivos. En González-Esteban, José Luis; García-Avilés, José Alberto (Eds.). Mediamorfosis. Radiografía de la innovación en el periodismo. Sociedad Española de Periodística, ISBN: 978-84-948233-5-0, pp. 123-136.

Sádaba, C. y Sánchez-Blanco, C. (2019). Los usos y preferencias informativas de los públicos: evasión informativa y exposición incidental; itinerarios, participación y redes sociales; radio, audio y podcast; publicidad y bloqueo de anuncios (ad-blocking). En: Toural-Bran, C. y LópezGarcía, X. Ecosistema de cibermedios en España. Tipologías, iniciativas, tendencias narrativas y desafios. Salamanca: Comunicación Social, pp. 109-140. ISBN: 978-84-17600-08-2.

Seyedghorban, Z., Tahernejad, H. y Matanda, M.J. (2016). Reinquiry into Advertising Avoidance on the Internet: A Conceptual Replication and Extension. Journal of Advertising, 45, 1, pp. 120129. https://doi.org/10.1080/00913367.2015.1085819

Singh, A. K. y Potdar, V. (2009). Blocking online advertising. A state of art. Proceedings Industrial Technology, ICIT 2009, IEEE International Conference, pp. 1-11.

Söllner, J. Y Dost, F. (2019). Exploring the Selective Use of Ad Blockers and Testing Banner Appeals to Reduce Ad Blocking. Journal of Advertising, 48, 3, pp. 302-312. https://doi.org/10.108 0/00913367.2019.1613699

Southgate, D. (2017). The emergence of Generation $\mathrm{Z}$ and its impact in advertising: Long-term implications for media planning and creative development. Journal of Advertising Research, 57, 2, pp. 227-235. https://doi.org/10.2501/JAR-2017-028

Speck, P.S. y Elliott, M.T. (1997). Predictors of Advertising Avoidance in Print and Broadcast Media. Journal of Advertising, 26, 3, pp. 61-76. https://doi.org/10.1080/00913367.1997.1067 3529

Tang, Ji, Zhang, P. y Wu, P. F.W. (2015). Categorizing consumer behavioral responses and artifact design features: The case of online advertising. Information Systems Frontiers, 17, 3, pp. 513-532. https://doi.org/10.1007/s10796-014-9508-3 
Tudoran, A. A. (2019). Why do internet consumers block ads? New evidence from consumer opinion mining and sentiment analysis. Internet Research, Vol. 29 No. 1, pp. 144-166. https://doi. org/10.1108/IntR-06-2017-0221

Van der Goot, M. J., Rozendaal, E., Opree, S.J.; Ketelaar, P.E. y Smit, E. G. (2018). Media generations and their advertising attitudes and avoidance: a six-country comparison. International Journal of Advertising, 37, 2, pp. 289-308. https://doi.org/10.1080/02650487.2016.1240469

Walbesser, J. L. (2011). Blocking advertising blocking: the war over internet advertising and the effect on intellectual property. Intellectual Property \& Technology Law Journal, 23, 1, pp. 19-26.

Zambrano, A. y Pickard, C. (2018). A defense of ad blocking and consumer inattention. Ethics and Information Technology, 20, pp.143-155. https://doi.org/10.1007/s10676-018-9454-8 